\title{
Current Challenges in the Development of Quantum Dot Sensitized Solar Cells
}

\author{
Iván Mora-Seró
}

Quantum dot sensitized solar cells (QDSSCs) have experienced a continuous performance growth in the past years presenting a photoconversion efficiency $>13 \%$. QDSSCs constitute a smart approach to take advantage of the properties of semiconductor quantum dots (QDs), mitigating the transport constrains. In contrast with other QD solar cell configurations, for QDSSCs, the record efficiencies have been reported with $\mathrm{Pb}$ and $\mathrm{Cd}$-free based sensitizers. The development of techniques in order to provide photoanodes with very high QD loading and the discovery of new electrolytes, including all solid configurations, are the most important future challenges that this technology must address to further increase cell performance and stability.

devices, including energy applications in photovoltaic devices. ${ }^{[6]}$ However, their application to the fabrication of photovoltaic devices is constrained mostly by carrier transport limitations.

The individuality of the QDs, represented by the nanocrystal surrounded by the capping ligand, confines the exciton in a small regions. This fact presents advantages for the development of light emitting diodes (LEDs). ${ }^{\left[{ }^{[4]}\right.}$ However, this structure hinders the charge transport properties. In the photovoltaic process two consecutive step are required. ${ }^{[7]}$ First, light has to be absorbed creating photogenerated carriers,

Reducing the semiconductor size to dimensions in the same order or lower than twice the exciton Bohr radius allows tuning the properties of the semiconductor material, being the control of the bandgap the most manifest feature..$^{[1]}$ This is the so-called quantum confinement regime, and the nanocrystals exhibiting it in the three space dimensions are also known as Quantum Dots (QDs). Semiconductor QDs are an easily tunable family of materials with excellent optoelectronic properties. They can be synthesized at relatively low temperature, even at room temperature, in contrast with their bulk counterparts. ${ }^{[2]}$ In addition, they can be processed by solution techniques potentially allowing a low cost fabrication process and their implementation in flexible devices. ${ }^{[3]}$ QDs, that are few nanometers in size, usually own excellent crystalline quality, especially colloidal QDs, but their inherently high surface to volume ratio provides a special role to the surface states that will influence the properties of devices fabricated with QDs as building blocks. ${ }^{[4]}$ In colloidal QDs, surface states are partially passivated by organic ligands anchored to the QD surface. The ligands influence not just the passivation, but also the interactions between QD-QD and between QDs and other materials. With appropriated QD synthesis and surface passivation is possible to decrease the nonradiative recombination pathways, increasing significantly the photoluminescence quantum yield (PLQY) in comparison with their bulk counterparts. ${ }^{[5]}$ These properties give QDs an immense potential for the development of optoelectronic

Prof. I. Mora-Seró

Institute of Advanced Materials (INAM)

Universitat Jaume I

Castellón de la Plana, Castelló 12071, Spain

E-mail: sero@uji.es

The ORCID identification number(s) for the author(s) of this article can be found under https://doi.org/10.1002/aenm.202001774.

DOI: 10.1002/aenm.202001774 splinting electron, and hole Fermi levels. However, a second step of charge separation at the selective contacts is necessary in order to convert the free energy harvested in the first step into usable work..$^{[7]}$ In QD solar cells, a light absorbing layer, formed by colloidal QDs as building blocks, is sandwiched between two different electron and hole selective contacts. ${ }^{[8]}$ In order to maximize the photogenerated power, all the incident light has to be absorbed by the QD layer, requiring QD films with at least several hundred nanometer thickness. At the same time, photogenerated electron and holes have to be transported through the QD layer to their respective selective contact, before they recombine. The presence of ligands between QDs, non-passivated trap states, and QD size distribution, causing a bandgap distribution where photogenerated carried can be trapped in low bandgap QDs, hinders carrier transport in colloidal QDs solar cells, as we discuss below.

An interesting alternative to this configuration is the QD Sensitized Solar Cell (QDSSC) structure, similar to the Dye Sensitized Solar Cells (DSSCs) but using semiconductor QDs instead of molecular dyes to sensitize a mesoporous electrode to visible light. ${ }^{[9]}$ In DSSCs, a molecular dye absorbs light and photogenerated electron and hole and quickly injected into two different transporting media. In conventional solar cells electron and holes are transported to the selective contacts through the same media in which both are photogenerated. Consequently, both kind of carriers cohabit in the same material until they are extracted at the selective contacts, increasing the probability of recombination. In contrast, in DSSCs just one kind of carrier is transported by each transporting media. As electron and hole transporting materials (ETM and HTM, respectively) just contain one kind of carrier, and consequently recombination is reduced, it allows to relax the high quality requirement of these materials, generally needed to reduce recombination. In addition, transport is mainly related to the individual properties of these materials rather than to the properties of the QD light 
absorber, permiting to bypass some of the transport limitation of QD thin films. In addition, to ensure the collection of the incident light in QDSSCs, a mesoporous electrode to increase the effective surface area, formed by nanoparticles (commonly of $\mathrm{TiO}_{2}$ ) with several tenths of nanometers size, are used as ETM. Eventually, the use of a redox couple in a liquid electrolyte guarantee the contact of mesoporous structure acting as HTM.

QDSSCs represent the triumph of an idea since their beginnings were not very promising. First report of the sensitization of nanoporous $\mathrm{TiO}_{2}$ electrodes with colloidal InP QDs showed photoconversion activity but with a very poor performance with external quantum efficiency (EQE) of $<1 \% .{ }^{[10]}$ Interestingly, 12 years later in 2010, the first experimental demonstration of internal quantum efficiency (IQE) higher than 100\%, in fact close to $200 \%$, taking advantage of the multiple exciton generation in PbS QDs, was reported for QDSSC configuration. ${ }^{[11]}$ One year later, the first demonstration of EQE > 100\% was reported for thin-film colloidal QD solar cell configuration. ${ }^{[12]}$

At the early stages of QDSSCs, this technology had to face two main challenges, that in fact although with different nuances they are the same ones that QDSSCs are currently facing: QD loading and device long term stability. At the beginning of this century, DSSC was a scientifically established technology, ${ }^{[13]}$ with a standard configuration formed by a mesoporous $\mathrm{TiO}_{2}$ photoanode, the sensitizer dye, a liquid electrolyte using iodine/iodide redox couple, and a platinized counter electrode to regenerate the redox couple extracting holes. In this established structure, the substitution of one part by another, i.e., the molecular dye by the semiconductor QD, does not change the concept but distorted the experimental feasibility. On the one hand, colloidal QDs with long molecular capping present sizes of the same range than the pores of the standard nanostructured electrode used, limiting the QD loading and consequently the light harvesting. On the other hand, most QDs are not stable in iodine/ iodide electrolyte, and new redox systems are required. At the same time, the change of redox couple also force the use of an appropriated counted electrode. In summary, the structure of QDSSCs has to be rethought with respect to DSSCs in order to increase the performance and the stability. ${ }^{[14]}$ For example, it was needed to use a new HTM in comparison with DSSCs, being the most widespread an aqueous electrolyte with polysulfide redox and brass counter electrode suited for this redox system. ${ }^{[15-17]}$

The strategy of growing directly the semiconductor QDs on the surface of the porous electrodes ${ }^{[18]}$ allowed to increase the QD loading. Chemical bath deposition $(\mathrm{CBD})^{[19]}$ or successive ionic layer adsorption and reaction (SILAR) ${ }^{[20]}$ permitted the growth of semiconductor QD on the surface of the mesoporous photoanodes. However, the crystal quality of the QDs grown by these methods is lower than the obtained for colloidal QDs and subsequent passivation is needed. ZnS passivation, ${ }^{[21]}$ that reduce the photocarrier recombination, ${ }^{[22]}$ has demonstrated a huge success in order to increase the performance of QDSSCs, not just in the case of QDs grown by CBD or SILAR but also for colloidal QDs. In fact this treatment is a standard step in most of the current QDSSCs, and QD passivation has been one of the most commonly used strategies in order to improve the performance of QDSSCs. ${ }^{[23,24]}$ The use of QDs directly grown on the mesoporous electrode allowed to increase the photoconversion efficiency (PCE) to $4-5 \% .{ }^{[25]}$
The easy preparation of QDSSCs by low demanding techniques as CBD and SILAR, where sensitization just require bath or dipping in simple solutions with the precursors, and also the lower cost in comparison with DSSCs (mostly due to the cost of precursors for QDs, redox, or counter electrode), popularized enormously QDSSCs despite at that stage the PCE remained significantly below the PCE reported for DSSCs. Probably QDSSCs are the solar cells more easy and accessible for preparation allowing many research group, even with limited resources, to start a research line on this topic. This fact allowed that many groups incorporated into the QDSSCs research pushing the interest and results of this field up.

However, the crystalline quality of QDs directly grown on the mesoporous electrode was limiting the QDSSC performance. QDSSCs prepared with colloidal QDs presented just slight lower performance than the ones prepared with QDs directly grown, despite the former presented much lower light absorption than the later, ${ }^{[26]}$ indicating the superior potentiality of colloidal QDs for the development of solar cell and the limitation arisen from the QD loading. Different techniques were investigated to sensitize mesoporous electrodes with colloidal QDs as direct absorption of as synthesized QDs or the use molecular likers to anchor the colloidal QDs to the photoanode surface. ${ }^{[17,27]}$ Zhong and co-workers developed capping-ligand-induced self-assembly (CLIS) method to increase the colloidal QD loading in sensitized electrodes, by pipetting the mercaptopropionic acid (MPA)capped core/shell CdS/CdSe colloidal QD aqueous solution onto the $\mathrm{TiO}_{2} \cdot{ }^{[28]}$ The increase of colloidal QD loading boosted for the first time the performance of colloidal QDSSCs beyond 5\%, but most importantly focuses the attention on colloidal QDs as sensitizer and all the subsequent performance records in QDSSCs have been obtained with colloidal QDs. ${ }^{[24]}$

Currently the performance of QDSSCs has surpassed 13\%, [29-31] in the same range than DSSCs, and the fight to increase the QD loading is still at the center of the development. Different groups have achieved an efficiency $>13 \%$. Zhong and co-workers reported the highest QDSSCs laboratory and certified efficiencies, 13.71\% and $13.49 \%$, respectively, taking advantage of a proper passivation of core/shell colloidal QDs. ${ }^{[31]}$ Moreover, efficiencies $>13 \%$ have been also obtained by increasing the QD loading with two consecutive QD depositions. ${ }^{[29,30]}$ The deposition of a surfactant after the colloidal QD sensitization allowed a secondary deposition of QDs, see Figure 1a, ${ }^{[29]}$ while a second deposition is also possible by sequential immersion in aqueous solution with the colloidal QDs, see Figure 1b. ${ }^{[30]}$ The co-sensitization has been a strategy also used in DSSCs, ${ }^{[32]}$ and in QDSSCs ${ }^{[33,34]}$ to extend the light harvesting range. Interestingly, also hybrid co-sensitization using QDs and dyes designed to link to the QDs have been developed. ${ }^{[35]}$ However, beyond the broadening of the light absorption range, a second cycle of deposition, even with the same QD type, can improve loading and cell performance. ${ }^{[29]}$

The current PCE records for QDSSCs has been obtained with $\mathrm{Zn}-\mathrm{Cu}-\mathrm{In}-\mathrm{Se}$ (ZCISe) and/or Zn-Cu-In-S (ZCIS) QDs. ${ }^{[29-31]}$ Despite, most of the first reports on QDSSCs were based on $\mathrm{Pb}$ or Cd containing materials (as CdS, CdSe, or $\mathrm{PbS}$ ), ${ }^{[25,28]}$ in contrast with other semiconductor solution processed photovoltaics, high performances have been attained with $\mathrm{Pb}$ and $\mathrm{Cd}$-free materials, as $\mathrm{CuInS}_{2}$, ${ }^{[36]} \mathrm{ZCIS},{ }^{[30,31]}$ or ZCISe. ${ }^{[29-31,37,38]}$ While thin-film colloidal QDs have been developed first based on $\mathrm{PbS}$ and $\mathrm{PbSe} \mathrm{QDs}^{\left[{ }^{[8]}\right.}$ 


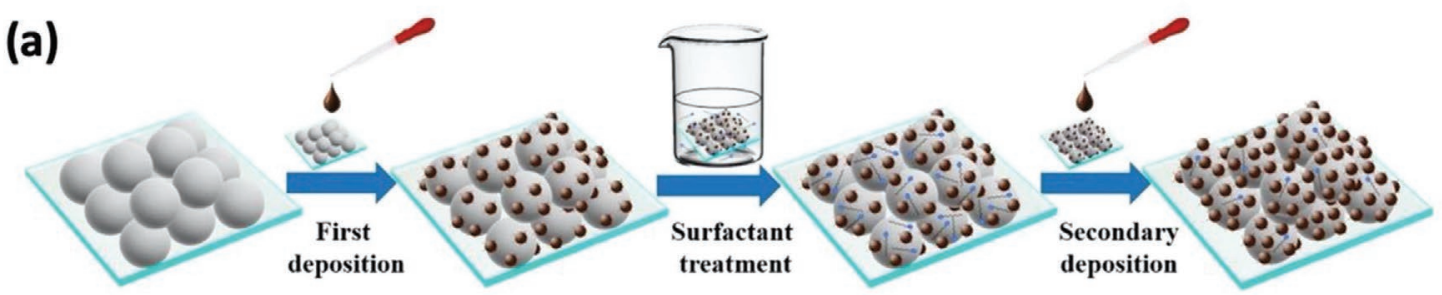

$\mathrm{TiO}_{2}$ electrode QD pre-sensitized
electrode
QD pre-sensitized electrode treated by surfactant
Electrode based on secondary deposition

$\mathrm{TiO}_{2}$
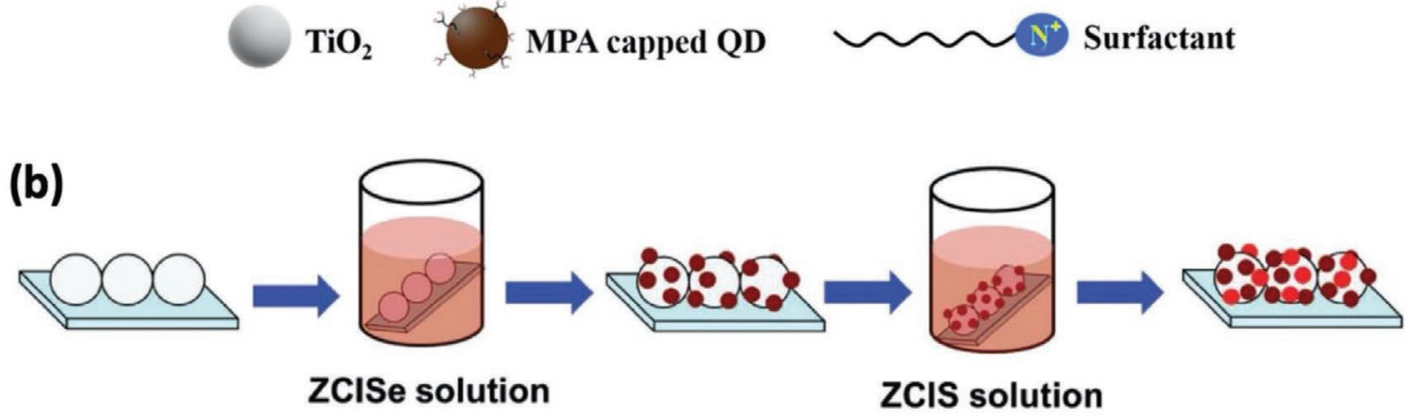

$\mathrm{TiO}_{2}$

- ZCISe QD

- ZCIS QD

Figure 1. Scheme of the sensitization procedure for double quantum dot deposition: a) using surface-ligand-assisted assemble. After a first deposition of QDs, photoanodes are treated by surfactant. Reproduced with permission. [29] Copyright 2019, American Chemical Society. b) Using a successive dipping in an aqueous solution at $50{ }^{\circ} \mathrm{C}$ containing QDs capped with 3-mercaptopropionic acid (MPA). Reproduced with permission. ${ }^{[30]}$ Copyright 2019, Wiley. This treatments allows to increase the QD loading after a second deposition of QDs.

and currently on lead halide perovskite QDs, with current performances $>16 \%,{ }^{[39]}$ in QDSSCs $\mathrm{Pb}$ and Cd-free QDs play currently a major role since most recent PCE records have been obtained with "green" QDs. This fact also represents an advantage with respect to the solution processed halide perovskite solar cells (PSCs), where PSCs presenting PCE exceeding the record for QDSSCs are always based on $\mathrm{Pb}$ halide perovskites. ${ }^{[40]}$ However, PSCs present outstanding record PCE $>25 \%$. $^{[41]}$

QDSSCs also present another interesting advantages. Very recently Klimov and co-workers have reported that in QDSSC configuration trapped photogenerated carriers can be partially recovered, and consequently recycled to ultimately contribute to the device efficiency. ${ }^{[38]}$ They compared QDSSCs sensitized with colloidal ZCISe QDs attached to the $\mathrm{TiO}_{2}$ using different capping providing them different binding interaction with $\mathrm{TiO}_{2}$ and different steric hindrance. They show that similar performance can be obtained with different capping providing both weak and strong binding interaction with $\mathrm{TiO}_{2}$, but lower performance was obtained for capping with strong steric hindrance as reduce the QD loading. Despite, in samples with weak binding interaction, most of the photogenerated carriers are trapped in QD shallow states, they can be later injected into the $\mathrm{TiO}_{2}$ conduction band, contributing to the final device performance, if the trap lay above (below) the ETM (HTM) conduction (valence) band, see Figure 2a. In the same line, QDSSCs have a good tolerance to QD size dispersion as bigger QDs, with narrower QDs, can still injecting into ETM
(HTM) if the conduction (valence) band lay above (below) the conduction band of the ETM (the redox level), see Figure 2b. This property confers an advantage of QDSSCs in comparison with thin-film colloidal QD solar cells where both traps and size distribution affect deleteriously the device transport properties, see Figure 2c. These result not just highlight the potentiality of QDSSCs to bypass the transport constrains arisen from traps and size distribution, but also point out again the increase of QD loading as a key aspect to improve QDSSC performance.

Beyond the QD loading, QDSSCs face a second key challenge, the device stability. This challenge has received significantly lower attention than the QD loading probably because QDSSCs did not present, at the first stages, enough performance and the research community focused first on this issue. However, nowadays when PCEs have surpassed the $13 \%$ in the same range than DSSCs, the stability of this devices requires more attention than historically received. Stability was the most important problem during the first steps of this technology as the iodine/iodide redox couple commonly used for DSSCs affects most of the semiconductors corroding them in few minutes. However, previous works pointed out that polysulfide redox provide longer stability to semiconductors in liquid electrolytes ${ }^{[16]}$ and this is the redox system that most extensively has been used in standard and in record QDSSCs. ${ }^{[24,29-31]}$ Despite, this redox system has allowed the QDSSCs evolution PCE from $<1 \%$ to $>13 \%$, it is far to be an optimum choice. While for sealed samples stored under dark ${ }^{[42]}$ or room light, ${ }^{[43]} 1000 \mathrm{~h}$ stability has been reported, for samples 
(a)

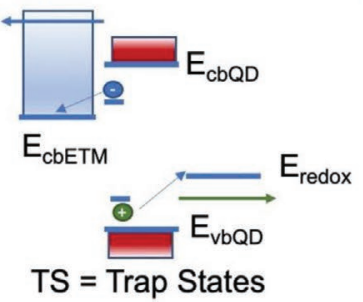

(b)
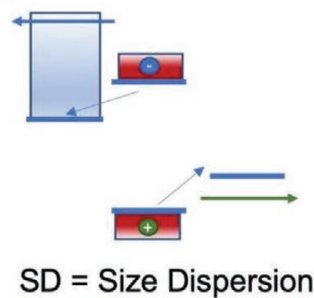

(c)
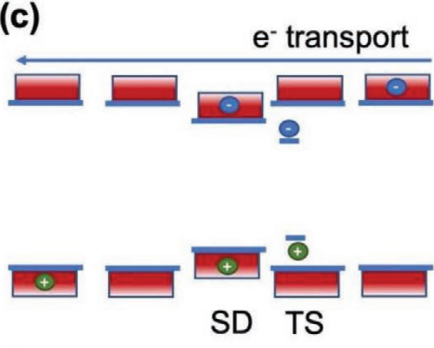

$\mathrm{h}^{+}$transport

Figure 2. In QDSSCs, generation and transport are decoupled in different materials. a) Energy level scheme of a QDSSC formed by the conduction and valence band of QD sensitizer, $E_{c b Q D}$ and $E_{v b Q D}$, respectively, the conduction band of the ETM, $E_{c b E T M}$, commonly TiO ${ }_{2}$, and the redox level, $E_{r e d o x}$, commonly polysulfide, acting as HTM. Part of the electron (hole) trapped at trap states situated below (above) $E_{c b Q D}\left(E_{v b Q D}\right.$ ), can be recovered as it has been recently shown, ${ }^{[38]}$ where injection is represented by thin blue arrows, and electrons (holes) are transported along the ETM (HTM), represented by thicker blue (green) arrows. b) QD size distribution, where bigger QDs present narrower bandgaps, also has a reduced effect in QDSSCs as long as $\mathrm{E}_{\mathrm{cbQD}}\left(\mathrm{E}_{\mathrm{vbQD}}\right)$ remain above (below) $\mathrm{E}_{\mathrm{cbETM}}\left(\mathrm{E}_{\mathrm{redox}}\right)$ and consequently can still injecting. $\mathrm{c}$ ) In contrast, in thin film colloidal QD solar cells both carriers are generated and transported along a thick layer of QDs. Photocarriers move from QD to QD until they arrive to the selective contacts. Carriers trapped in surface states or even in bigger QDs (lower bandgap) do not contributed to the photocurrent until they are released again to the bands, meanwhile they can recombine and be lost for the photoconversion process.

analyzed under 1 sun illumination there is no report ${ }^{[24]}$ of negli-

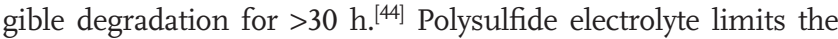
long-term stability via oxidation of the QDs. ${ }^{[38]}$ In addition, the oxidation of $\mathrm{Cu}$ in the brass electrode, also extensively used in combination with polysulfide system, ${ }^{[16]}$ upward the redox level, limiting the open circuit potential, $\mathrm{V}_{\text {oc }}{ }^{[38]}$ While carbon electrodes have provide interesting results in terms of efficiency, ${ }^{[34,37,38]}$ the stability in combination with polysulfide is severely limited. ${ }^{[38]}$ Consequently, the future applicability of QDSSCs will depend on getting a compatible semiconductor $\mathrm{QD}$, redox, and counter electrode system stable beyond polysulfide.
An interesting alternative could be all-solid devices. However, in this case performance is limited, as just thin electrodes, $<2 \mu \mathrm{m}$, can be employed to avoid hole transport loses in the solid HTM, ${ }^{[45]}$ in comparison with the $\approx 30 \mu \mathrm{m}$ thick mesoporous photoanodes used in liquid QDSSCs. ${ }^{[29]}$ Thinner electrodes undoubtedly would limit the QD loading but still interesting for other applications as semitransparent or colored solar cells. Very recently it has been reported PCE $>7 \%$ for allsolid QDSSCs using $\mathrm{CH}_{3} \mathrm{NH}_{3} \mathrm{PbI}_{3}$ perovskite as sensitizer, ${ }^{[46]}$ see Figure 3a. In fact, the first report on halide perovskite for solar cell applications was with QDSSC configuration. ${ }^{[47]}$ By the (a)

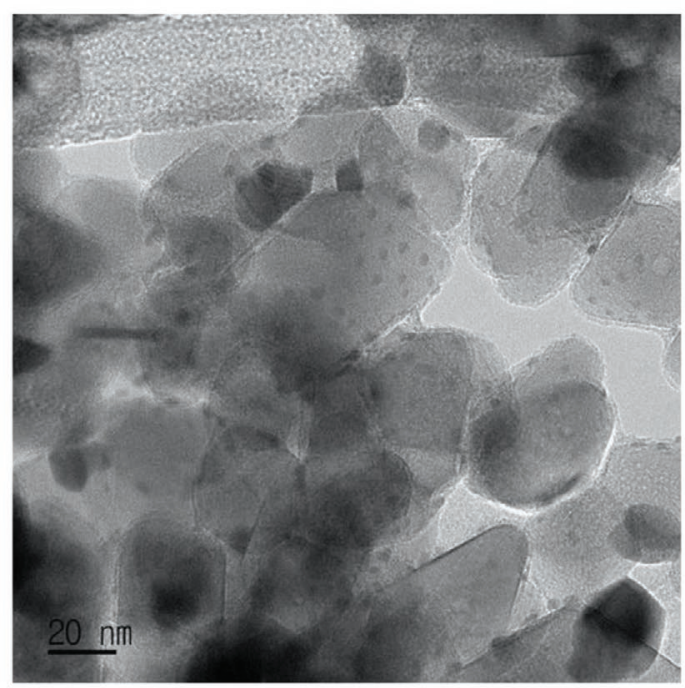

(b)
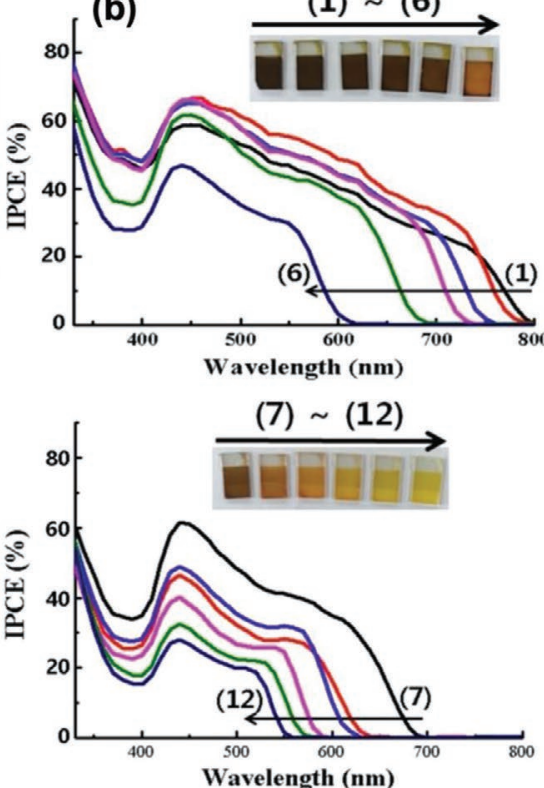

Figure 3. a) Transmission electron microscopy of a mesoporous $\mathrm{TiO}_{2}$ electrode sensitized with nanoscale $\mathrm{CH}_{3} \mathrm{NH}_{3} \mathrm{Pbl}_{3}$. Reproduced with permission. ${ }^{[48]}$ Copyright 2019, Elsevier. b) Incident photon to electron efficiency for QDSSC using different nanoscale $\mathrm{CH}_{3} \mathrm{NH}_{3} \mathrm{Pbl}_{x} \mathrm{Br}_{3-\mathrm{x}}$ perovskite as sensitizer, different curves correspond to photoanodes that have been sensitized with a perovskite with a iodine content progressively decreasing, from, from (1) with $x=3$ to (12) with $x=0$, Reproduced with permission. ${ }^{[46]}$ Copyright 2020, Wiley. 
control of the precursor concentration, it is possible to deposit halide perovskite QDs decorating the mesoporous $\mathrm{TiO}_{2}$ surface, see Figure 3a. It has been demonstrated that in this conditions, without coalescence between the perovskite nanoparticles, the system using spiro-OMeTAD as HTM behaves as a classic QDSSC. ${ }^{[46,48]}$ All-solid perovskite QDSSCs are semitransparent but presenting a relatively high PCE, in addition it is possible to tune the color of the device by composition engineering, for example, changing the ratio of I and $\mathrm{Br}$ halide, see Figure 3b, ${ }^{[46]}$ providing a broad range of design possibilities for building integration and indoor applications. In this way, all-solid QDSSCs can take advantage of the intensive work regarding the stability that is being done in the halide perovskite field.

In summary, QDSSCs are an excellent examples of the power of a focused research, starting from a concept with practically negligible photoconversion efficiency until achieve efficiencies beyond $13 \%$ in the same range as other solution-processed solar cells such as DSSCs. Despite the huge progress of the QDSSCs field in the last years, this technology face important challenges regarding a future commercialization. The most important ones are the increase of the QD loading and device stability. The increase of QD loading will help to increase the device efficiency but also to reduce the thickness of the sensitized electrode that favors the design of all-solid devices that can help also to increase stability. In addition, to increase the device stability, it seems mandatory to find an alternative to polysulfide redox, which not only improves stability, but also reduces the energy position of the redox level to increase $\mathrm{V}_{\text {oc }}$. Moreover, a compatible counter electrode will be also necessary. This effort will allow to harness the advantages of QDSSCs in comparison with other solution-processed solar cells, as the improved transport properties, with a reduced effect of traps and QD size distribution, and the lower toxicity, as record performance in QDSSCs have been obtained with "green" QDs, $\mathrm{Pb}$ and Cd-free. Consequently, there is room for further improvements in this photovoltaic technology in the near future.

\section{Acknowledgements}

Financial support from the European Research Council (ERC) via Consolidator Grant (724424-No-LIMIT) and Generalitat Valenciana via Prometeo Grant Q-Devices (Prometeo/2018/098) is gratefully acknowledged.

\section{Conflict of Interest}

The author declares no conflict of interest.

\section{Keywords}

perovskites, photoconversion efficiency, quantum dots, solar cells, solar cell stability

Received: May 28, 2020

Revised: July 2, 2020

Published online: July 26, 2020
[1] A. P. Alivisatos, Science 1996, 271, 933

[2] Y. Pu, F. Cai, D. Wang, J.-X. Wang, J.-F. Chen, Ind. Eng. Chem. Res. 2018, 57, 1790.

[3] M. Graetzel, R. A. J. Janssen, D. B. Mitzi, E. H. Sargent, Nature 2012, 488, 304.

[4] A. P. Litvin, I. V. Martynenko, F. Purcell-Milton, A. V. Baranov, A. V. Fedorov, Y. K. Gun'ko, J. Mater. Chem. A 2017, 5, 13252.

[5] J. Owen, L. Brus, J. Am. Chem. Soc. 2017, 139, 10939.

[6] H. Lu, Z. Huang, M. S. Martinez, J. C. Johnson, J. M. Luther, M. C. Beard, Energy Environ. Sci. 2020.

[7] J. Bisquert, D. Cahen, G. Hodes, S. Rühle, A. Zaban, J. Phys. Chem. B 2004, 108, 8106.

[8] G. H. Carey, A. L. Abdelhady, Z. Ning, S. M. Thon, O. M. Bakr, E. H. Sargent, Chem. Rev. 2015, 115, 12732.

[9] B. O' Regan, M. Grätzel, Nature 1991, 353, 737.

[10] A. Zaban, O. I. Micic, B. A. Gregg, A. J. Nozik, Langmuir 1998, 14, 3153.

[11] J. B. Sambur, T. Novet, B. A. Parkinson, Science 2010, 330, 63.

[12] O. E. Semonin, J. M. Luther, S. Choi, H.-Y. Chen, J. Gao, A. J. Nozik, M. C. Beard, Science 2011, 334, 1530

[13] A. Hagfeldt, M. Grätzel, Accounts Chem. Res. 2000, 33, 269.

[14] I. Mora-Seró, J. Bisquert J. Phys. Chem. Lett. 2010, 1, 3046.

[15] I. Mora-Seró, S. Giménez, T. Moehl, F. Fabregat-Santiago, T. Lana-Villareal, R. Gómez, J. Bisquert, Nanotechnology 2008, 19, 424007.

[16] G. Hodes, J. Manassen, D. Cahen, J. Electrochem. Soc. 1980, 127, 544.

[17] S. Giménez, I. Mora-Seró, L. Macor, N. Guijarro, T. Lana-Villarreal, R. Gómez, L. J. Diguna, Q. Shen, T. Toyoda, J. Bisquert, Nanotechnology 2009, 20, 295204.

[18] a) R. Vogel, P. Hoyer, H. Weller, J. Chem. Phys. 1994, 98, 3183; b) R. Vogel, K. Pohl, H. Weller, Chem. Phys. Lett. 1990, 174, 241.

[19] a) L. J. Diguna, Q. Shen, J. Kobayashi, T. Toyoda, Appl. Phys. Lett. 2007, 91, 023116; b) O. Niitsoo, S. K. Sarkar, C. Pejoux, S. Rühle, D. Cahen, G. Hodes, J. Photochem. Photobiol. A: Chem. 2006, 181, 306.

[20] H. J. Lee, M. Wang, P. Chen, D. R. Gamelin, S. M. Zakeeruddin, M. Grätzel, M. K. Nazeeruddin, Nano Lett. 2009, 9, 4221.

[21] a) Q. Shen, J. Kobayashi, L. J. Diguna, T. Toyoda, J. Appl. Phys. 2008, 103, 084304; b) S. Hachiya, Q. Shen, T. Toyoda, J. Appl. Phys. 2012, $111,104315$.

[22] I. Mora-Seró, S. Giménez, F. Fabregat-Santiago, R. Gómez, Q. Shen, T. Toyoda, J. Bisquert, Accounts Chem. Res. 2009, 42, 1848.

[23] Z. Ren, J. Wang, Z. Pan, K. Zhao, H. Zhang, Y. Li, Y. Zhao, I. Mora-Sero, J. Bisquert, X. Zhong, Chem. Mater. 2015, 27, 8398.

[24] Z. Pan, H. Rao, I. Mora-Seró, J. Bisquert, X. Zhong, Chem. Soc. Rev. 2018, 47, 7659.

[25] a) V. Gonzalez-Pedro, C. Sima, G. Marzari, P. P. Boix, S. Gimenez, Q. Shen, T. Dittrich, I. Mora-Sero, Phys. Chem. Chem. Phys. 2013, 15, 13835; b) P. K. Santra, P. V. Kamat, J. Am. Chem. Soc. 2012, 134, 2508; c) Q. Zhang, X. Guo, X. Huang, S. Huang, D. Li, Y. Luo, Q. Shen, T. Toyoda, Q. Meng, Phys. Chem. Chem. Phys. 2011, 13, 4659.

[26] a) S. Giménez, X. Xu, T. Lana-Villarreal, R. Gómez, S. Agouram, Muñoz-Sanjosé, I. Mora-Seró, J. Appl. Phys. 2010, 108, 064310; b) E. Martínez-Ferrero, I. Mora-Seró, J. Alberoa, S. Giménez, J. Bisquert, E. Palomares, Phys. Chem. Chem. Phys. 2010, 12, 2819.

[27] a) N. Guijarro, T. Lana-Villarreal, I. Mora-Seró, J. Bisquert, R. Gómez, J. Phys. Chem. C 2009, 113, 4208; b) I. Robel, V. Subramanian, M. Kuno, P. V. Kamat, J. Am. Chem. Soc. 2006, 128, 2385.

[28] Z. Pan, H. Zhang, K. Cheng, Y. Hou, J. Hua, X. Zhong, ACS Nano 2012, 6, 3982

[29] W. Wang, L. Zhao, Y. Wang, W. Xue, F. He, Y. Xie, Y. Li, J. Am. Chem. Soc. 2019, 141, 4300.

[30] Z. Pan, L. Yue, H. Rao, J. Zhang, X. Zhong, Z. Zhu, A. K. Y. Jen, Adv. Mater. 2019, 31, 1903696. 
[31] H. Rao, M. Zhou, Z. Pan, X. Zhong, J. Mater. Chem. A 2020, 8, 10233.

[32] A. Yella, H.-W. Lee, H. N. Tsao, C. Yi, A. K. Chandiran, M. K. Nazeeruddin, E. W.-G. Diau, C.-Y. Yeh, S. M. Zakeeruddin, M. Grätzel, Science 2011, 334, 629.

[33] a) P. K. Santra, P. V. Kamat, J. Am. Chem. Soc. 2013, 135, 877; b) D. Esparza, T. Lopez-Luke, J. Oliva, A. Cerdán-Pasarán, A. Martínez-Benítez, I. Mora-Seró, E. D. I. Rosa, Electrochim. Acta 2017, 247, 899.

[34] W. Wang, W. Feng, J. Du, W. Xue, L. Zhang, L. Zhao, Y. Li, X. Zhong, Adv. Mater. 2018, 30, 1705746.

[35] V. M. Blas-Ferrando, J. Ortiz, V. González-Pedro, R. S. Sánchez, I. Mora-Seró, F. Fernández-Lázaro, Á. Sastre-Santos, Adv. Funct. Mater. 2015, 25, 3220.

[36] Z. Pan, I. Mora-Seró, Q. Shen, H. Zhang, Y. Li, K. Zhao, J. Wang, X. Zhong, J. Bisquert, J. Am. Chem. Soc. 2014, 136, 9203.

[37] a) J. Du, Z. Du, J.-S. Hu, Z. Pan, Q. Shen, J. Sun, D. Long, H. Dong L. Sun, X. Zhong, L.-J. Wan, J. Am. Chem. Soc. 2016, 138, 4201; b) S. Jiao, J. Du, Z. Du, D. Long, W. Jiang, Z. Pan, Y. Li, X. Zhong, J. Chem. Phys. Lett. 2017, 8, 559.

[38] J. Du, R. Singh, I. Fedin, A. S. Fuhr, V. I. Klimov, Nat. Energy 2020, 5,409
[39] M. Hao, Y. Bai, S. Zeiske, L. Ren, J. Liu, Y. Yuan, N. Zarrabi, N. Cheng, M. Ghasemi, P. Chen, M. Lyu, D. He, J.-H. Yun, Y. Du, Y. Wang, S. Ding, A. Armin, P. Meredith, G. Liu, H.-M. Cheng, L. Wang, Nat. Energy 2020, 5, 79.

[40] A. K. Jena, A. Kulkarni, T. Miyasaka, Chem. Rev. 2019, 119, 3036.

[41] M. A. Green, E. D. Dunlop, J. Hohl-Ebinger, M. Yoshita, N. Kopidakis, A. W. Y. Ho-Baillie, Prog. Photovolt: Res. Appl. 2020, $28,3$.

[42] X. Zhang, X. Huang, Y. Yang, S. Wang, Y. Gong, Y. Luo, D. Li, Q. Meng, ACS Appl. Mater. Interfaces 2013, 5, 5954.

[43] Y. Yang, L. Zhu, H. Sun, X. Huang, Y. Luo, D. Li, Q. Meng, ACS Appl. Mater. Interfaces 2012, 4, 6162.

[44] J. Du, X. Meng, K. Zhao, Y. Li, X. Zhong, J. Mater. Chem. A 2015, 3 , 17091.

[45] F. Fabregat-Santiago, J. Bisquert, L. Cevey, P. Chen, M. Wang, S. M. Zakeeruddin, M. Grätzel, J. Am. Chem. Soc. 2009, 131, 558.

[46] S.-M. Yoo, S.-Y. Lee, E. Velilla Hernandez, M. Kim, G. Kim, T. Shin, M. K. Nazeeruddin, I. Mora-Seró, H. J. Lee, ChemSusChem 2020, $n / a$

[47] A. Kojima, K. Teshima, Y. Shirai, T. Miyasaka, J. Am. Chem. Soc. 2009, 131, 6050

[48] S.-M. Yoo, S. J. Yoon, J. A. Anta, H. J. Lee, P. P. Boix, I. Mora-Seró, Joule 2019, 3, 2535.

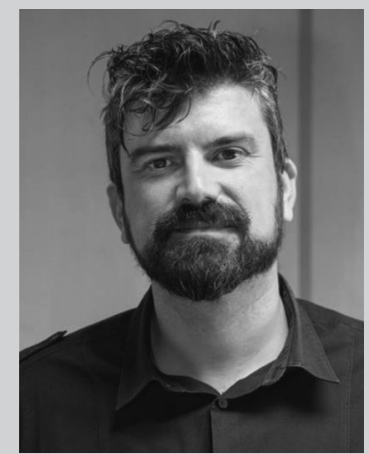

Iván Mora-Seró (Ph.D. Physics 2004) is researcher at Universitat Jaume I de Castelló (Spain). His research is focused on crystal growth, nanostructured devices, transport and recombination properties, photocatalysis, characterization and development of photovoltaic and optoelectronic devices. Recent research activities are focused on new concepts for photovoltaic conversion and light emission (LEDs and light amplifiers) based on nanoscaled devices and semiconductor materials following two main lines: semiconductor quantum dots and halide perovskites. 